\title{
Peligro geológico, susceptibilidad y riesgo de desastre en la cuenca inambari
}

\section{Geological hazard, susceptibility and disaster risk in the inambari watershed}

DOI: $10.46932 /$ sfjdv2n4-059

Received in: May 1st, 2021

Accepted in: Jun 30th, 2021

\author{
Newton Machaca \\ Docente Principal, Facultad de Ingeniería Geológica y Metalúrgica, UNA-Puno, Perú, \\ E-mail: newtonmachaca@unap.edu.pe
}

Elier Pacheco

Investigador Agregado en el Instituto de Geofísica y Astronomía, Dpto. de Estudios Geoambientales, CITMA, Ciudad de la Habana, Cuba,

E-mail: elier@iga.com

\section{Dante Salas}

Docente Principal, Facultad de Ingeniería Geológica y Metalúrgica, UNA-Puno, Perú, E-mail: newtonmachaca@unap.edu.pe

Juvenal Medina

Jefe del Centro Nacional de Estimación, Prevención y Reducción del Riesgo de Desastres

(CENEPRED)

E-mail: jmedina@cenepred.gob.pe

\section{Dulio Gomez}

Geología Ambiental y Riesgos Geológicos-INGEMMET

E-mail: dgomez@ingemmet.gob.pe

\section{RESUMEN}

Se trata la susceptibilidad al peligro por movimientos de masas de tierra en zonas de geomorfología compleja como la cuenca Inambari y el problema se dirige a conocer cuáles son las característica que inducen a la ocurrencia de deslizamientos en la cuenca Inambari, el objetivo es obtener un documento que explique, que provocan estos movimientos y para esto se adecuó una metodología donde se emplean principalmente métodos de estudio que determinan la peligrosidad, vulnerabilidad y el nivel de riesgo de los deslizamientos, la metodología de investigación es científica cualitativa y de carácter básico aplicado con técnicas computarizadas y procedimientos matemáticos-estadísticos que en la práctica se materializan con el monitoreo y la comprobación de fotointerpretación lo que determinó la valoración de las zonas susceptibles del medio físico al peligro. Se obtienen resultados científicos con relación a la tolerancia de los procesos y a la susceptibilidad. Otros de carácter socioeconómico y cultural se basan en el establecimiento de un Sistema de Alerta Temprana.

Palabras Clave: cuenca, deslizamiento, Inambari, peligro geológico, riesgo de desastre

\begin{abstract}
It is about the susceptibility to danger by movements of earth masses in areas of complex geomorphology as the Inambari basin and the problem is directed to know what are the characteristics that induce the occurrence of landslides in the Inambari basin, the objective is to obtain a document that explains, which
\end{abstract}




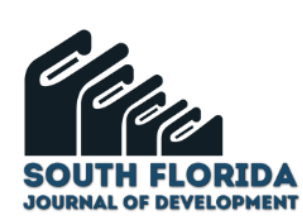

cause these movements and for this a methodology was adapted where study methods are mainly used to determine the danger, vulnerability and risk level of landslides, the research methodology is qualitative scientific and basic applied with computerized techniques and mathematical-statistical procedures that in practice are materialized with monitoring and photo-interpretation verification, which determined the assessment of susceptible areas of the physical environment to the danger. Scientific results are obtained in relation to the tolerance of the processes and susceptibility. Other socioeconomic and cultural results are based on the establishment of an Early Warning System.

Key words: basin, landslide, Inambari, geological hazard, disaster risk.

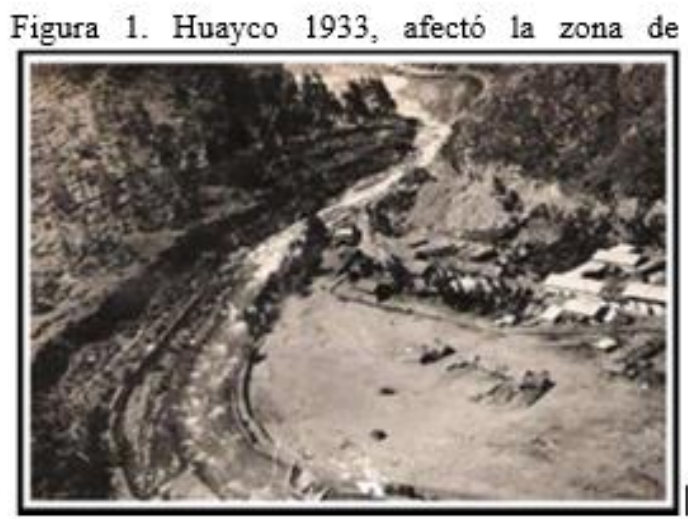

Figura 2. Sandia, población actual

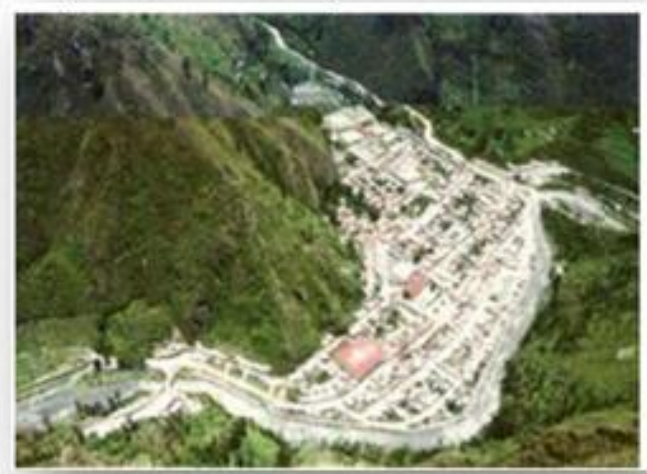

Figura 1. Mapa de ubicación Cuenca Inambari

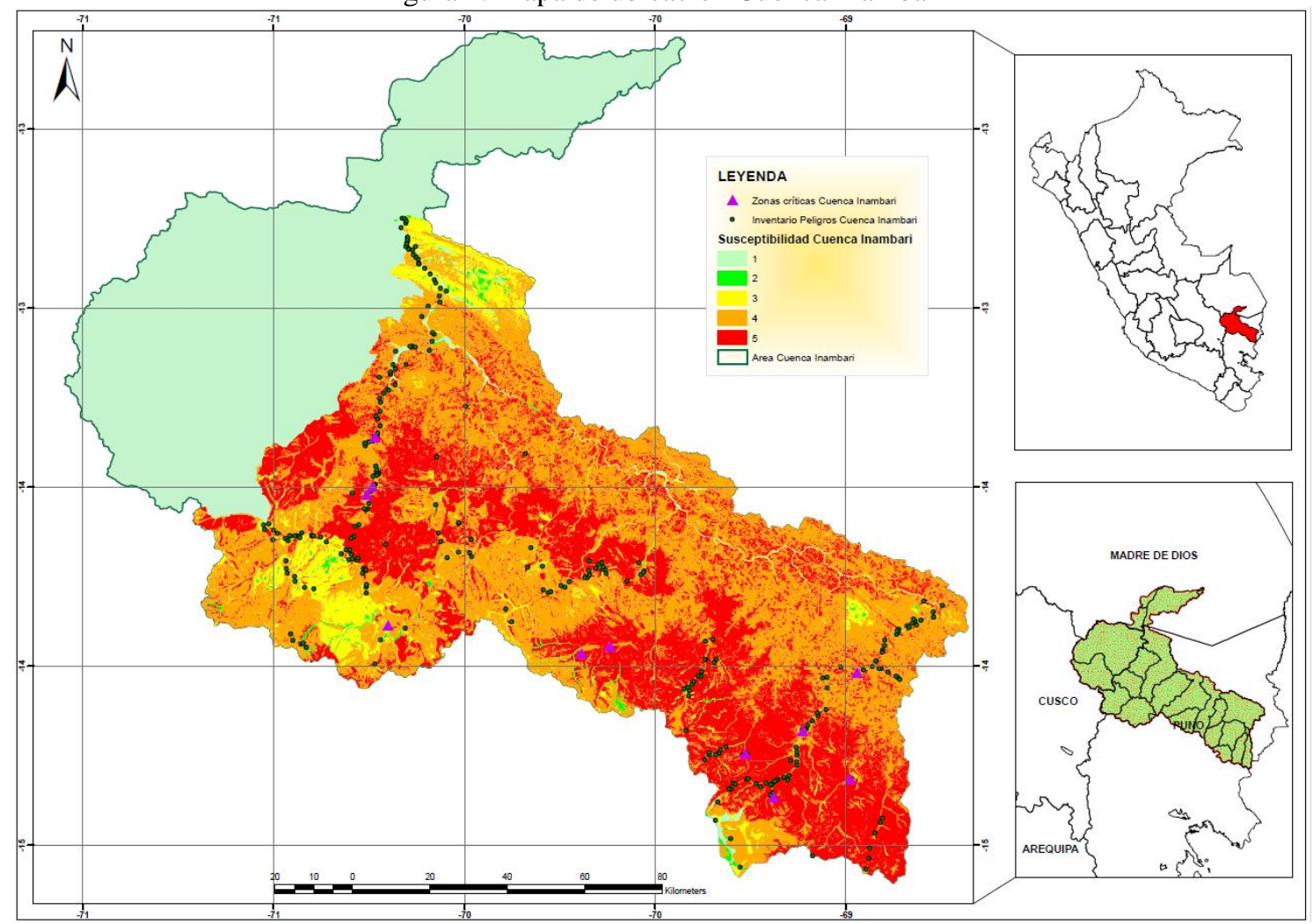




\section{INTRODUCCIÓN}

En las últimas décadas se han desarrollado numerosas estrategias para la gestión del riesgo por movimientos en masa. Por lo tanto, esperamos que el enfoque se utilice para modelar el riesgo de deslizamientos sociales en otras áreas geográficas para las que se dispone de información adecuada, y para modelar las consecuencias fatales de otros peligros. (Rossi, Guzzetti, Salvati, Donnini, \& Napolitano, 2019). El diseño, la implementación, la gestión y la verificación de los sistemas de alerta temprana de deslizamientos de tierra (LEWS) están ganando cada vez más atención en la literatura y entre los funcionarios del gobierno, los tomadores de decisiones y el público. (Guzzetti et al., 2020). Con el surgimiento del concepto de resiliencia, los académicos y profesionales han prestado cada vez más atención a la ciudad resiliente, que se ha convertido en un nuevo medio para hacer frente a los peligros. (Liu \& Song, 2020). Las soluciones basadas en la naturaleza (NbS) se destacan en acuerdos internacionales como el Marco de Sendai para la Reducción del Riesgo de Desastres 2015-2030 como estrategias prometedoras para reducir el riesgo de desastres, adaptarse al cambio climático y fortalecer la resiliencia comunitaria. (Arce-mojica, Nehren, Sudmeier-rieux, Julio, \& Anhuf, 2019). Involucrar a la comunidad en los sistemas de alerta temprana (EWS, por sus siglas en inglés) juega un papel esencial en salvar vidas, reducir lesiones y limitar el daño ambiental asociado con los desastres. (Sufri, Dwirahmadi, Phung, \& Rutherford, 2020).

\section{OBJETIVOS}

Determinar el peligro geológico por deslizamientos en la cuenca Inambari

Determinar el análisis de la susceptibilidad por movimientos en masa en la cuenca Inambari.

Determinar el riesgo de desastre la por movimientos en masa en la cuenca Inambari.

\section{ANTECEDENTES}

La cuenca Inambari, comprende territorios donde convergen una serie de fenómenos naturales y que han dado lugar a desastres con muchos daños y pérdidas. Eventos climatológicos como lluvias intensas en todo su territorio, nevadas, heladas y vientos fuertes entre otros en su zona altoandina. En enero del 2005 en el cerro Llamanipata localizado en el distrito de Sandia, se deslizó una gran parte existiendo el riesgo que todo el cerro se deslice, lo cual podría provocar un represamiento del río y por ende una inundación en el pueblo de Sandia y comunidades localizadas río abajo.

A pesar de este deslizamiento y las gestiones realizadas por el alcalde ante el INDECI y otros organismos, aún no produjo la declaratoria de emergencia. Frente a ese panorama y ante las gestiones del 
alcalde provincial, OXFAM GB dispuso dar soporte técnico en acciones de preparativos para desastres a través de PREDES.

OXFAM GB, en marzo del año 2005, financió la I Misión Técnica a Sandia, en la que se observó la amenaza existente y las vulnerabilidades del Comité de Defensa Civil y la población. Al realizarse un simulacro se determinó la no existencia de un Sistema de Alerta Temprana ante inundaciones, esto dio lugar a que PREDES elaborara el proyecto Misión Técnica II a Sandia, el cual comprende la Organización e Implementación de un SAT ante inundaciones, el mismo fue presentado y aprobado por OXFAM GB.

En este contexto PREDES y OXFAM GB vienen organizando e implementando un Sistema de Alerta Temprana que comprende la organización de un sistema de monitoreo y Vigilancia, la organización e implementación de un Sistema de Comunicaciones, la implementación de un Sistema de Alarma y la elaboración e implementación de un Plan de Evacuación.

\section{METODOLOGÍA}

La presente constituye un resultado de la investigación que fue sustentada sobre la base experimental de varios estudios realizados por el autor que han sido publicados en artículos y eventos de ámbito nacional e internacional con la intensión de guiar metodológicamente el desarrollo de las investigaciones relacionadas con movimientos en masa en los valles interandinos.

Las etapas de investigación que originaron la metodología son las siguientes:

- Diagnóstico para el manejo del riesgo.

- Análisis de la susceptibilidad y su influencia en la dinámica de los procesos por movimientos en masa.

- Elaboración de mapas temáticos.

- Estimación probabilística de pérdidas humanas y materiales

- Recomendaciones para mitigar las consecuencias.

\section{RESULTADOS Y DISCUSIÓN}

\section{- Peligro}

Dado que el peligro es un evento físico, potencialmente perjudicial y propio del lugar se obtuvo el siguiente resultado:

Tabla 1. Resultado del análisis de peligro, vulnerabilidad y riesgo de la cuenca Inambari Peligro vulnerabilidad y riesgo por movimientos en masa en la cuenca Inambari

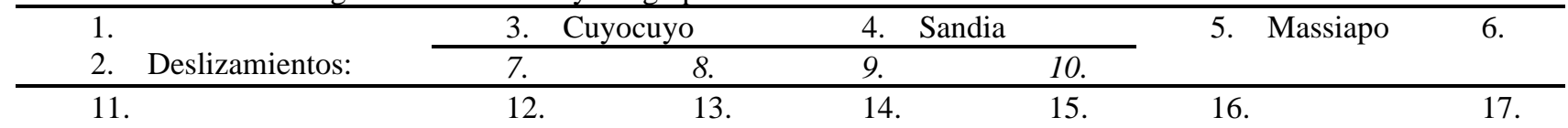




$\begin{array}{lllllll}\text { 18. Peligro } & 19.3 & 20 . & 21.4 & 22 . & 23.4 & 24 . \\ \text { 25. } & 26 . & 27 . & 28 . & 29 . & 30 . & 31 . \\ \text { 32. Vulnerabilidad } & 33.4 & 34 . & 35.5 & 36 . & 37.4 & 38 . \\ \text { 39. } & 40 . & 41 . & 42 . & 43 . & 44 . & 45 . \\ \text { 46. Riesgo } & 47.12 & 48 . & 49.20 & 50 . & 51.16 & 52 .\end{array}$

\section{- Susceptibilidad}

El análisis de la susceptibilidad a movimientos en masa de la cuenca Inambari se sustenta en un Sistema de Información Geográfica (SIG) que incluye: un inventario de deslizamientos, adquisición de datos espaciales, obtención del mapa de susceptibilidad y la evaluación de la precisión de los resultados.

Figura 2. Mapa de susceptibilidad de la cuenca Inambari

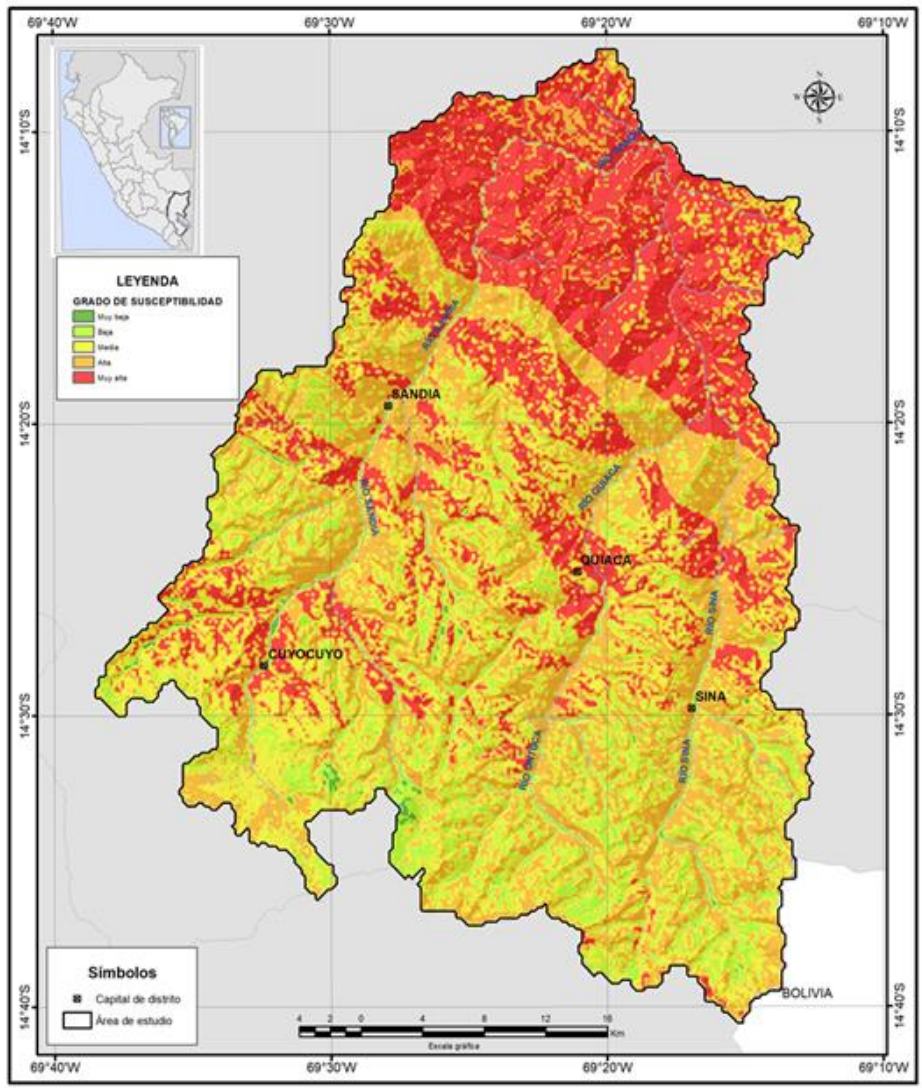

\section{- Riesgo}

La evaluación del nivel de riesgo es un procedimiento técnico que permite: analizar los parámetros de evaluación del fenómeno, factores condicionantes y la susceptibilidad de los mismos, analizar la vulnerabilidad de los elementos expuestos al peligro en función a los factores expuestos, la fragilidad y resiliencia, determinar y zonificar los niveles de riesgos y establecer medidas de control del riesgo 
de desastre. La gestión prospectiva del riesgo se desarrolló en función de la estimación y prevención, lo que desemboca en la evaluación del riesgo propiamente dicho.

La gestión correctiva del riesgo se desarrolla en función de la reducción del riesgo y la prevención del mismo lo cual se utilizará con fines de reasentamiento de la población afectada.

Los estudios de riesgo permiten identificar las zonas más expuestas frente a los movimientos en masa, toda esta información se utiliza como parte del sustento técnico-científico de la fase de diagnóstico, en la elaboración de los planes de prevención y reducción del riesgo de desastres, así como para los planes de reasentamiento.

Los instrumentos técnicos que se obtienen de la evaluación del riesgo son relevantes por que sirven para evitar las ocupaciones territoriales que generarían riesgo, por ejemplo, al asignar zonas de tratamiento paisajístico o especial, sirven también, para priorizar acciones que se deben cumplir para reducir el riesgo hacia un nivel aceptable, orientado a reasentamientos o desarrollo de medidas de control de riesgo; para prevenir la generación de nuevos riesgos y/o reducir el nivel de riesgo existente en áreas geográficas urbanizadas y como sustento de los planes integrales de áreas urbanas cuyas condiciones ameritan un tratamiento integral especial.

\section{CONCLUSIONES}

Según la metodología descrita, se concluye que los peores escenarios de riesgo por movimientos en masa en la cuenca Inambari son el represamiento del río Sandia por deslizamiento de las paredes del valle y el desagüe violento de la laguna que se forme, produciendo inundaciones graves en los principales poblados.

\section{AGRADECIMIENTOS}

Los autores agradecen la colaboración de personas e instituciones que han colaborado en esta publicación. 


\section{REFERENCIAS}

Arce-mojica, T. D. J., Nehren, U., Sudmeier-rieux, K., Julio, P., \& Anhuf, D. (2019). International Journal of Disaster Risk Reduction Nature-based solutions $(\mathrm{NbS})$ for reducing the risk of shallow landslides : Where do we stand? International Journal of Disaster Risk Reduction, 41(April), 101293. https://doi.org/10.1016/j.ijdrr.2019.101293

Guzzetti, F., Gariano, S. L., Peruccacci, S., Brunetti, M. T., Marchesini, I., Rossi, M., \& Melillo, M. (2020). Earth-Science Reviews Geographical landslide early warning systems. Earth-Science Reviews, 200(July 2019), 102973. https://doi.org/10.1016/j.earscirev.2019.102973

Liu, W., \& Song, Z. (2020). Review of studies on the resilience of urban critical infrastructure networks. Reliability Engineering and System Safety, 193(June 2019), 106617. https://doi.org/10.1016/j.ress.2019.106617

Rossi, M., Guzzetti, F., Salvati, P., Donnini, M., \& Napolitano, E. (2019). Earth-Science Reviews A predictive model of societal landslide risk in Italy. Earth-Science Reviews, 196(April), 102849. https://doi.org/10.1016/j.earscirev.2019.04.021

Sufri, S., Dwirahmadi, F., Phung, D., \& Rutherford, S. (2020). Progress in Disaster Science Review article A systematic review of Community Engagement (CE) in Disaster Early Warning Systems (Es). Progress in Disaster Science, 5, 100058. https://doi.org/10.1016/j.pdisas.2019.100058 